\title{
NEW INSIGHT INTO THE FISSION PROCESS FROM EXPERIMENTS WITH RELATIVISTIC HEAVY-ION BEAMS
}

A. KELIC, M. V. RICCIARDI, K.-H. SCHMIDT

Gesellschaft für Schwerionenforschung, GSI, 64291 Darmstadt, Planckstr. 1

\begin{abstract}
The results from a series of experiments performed with a novel inverse-kinematics approach using the experimental installations of GSI, Darmstadt, gave a new global insight into the nuclide production in fission reactions. Combined with previous results, this large body of data has been used to develop a new semi-empirical macroscopic-microscopic fission model.
\end{abstract}

\section{Introduction}

Several years ago, a research program has been initiated for studying the nuclear fission process with a new experimental approach by using relativistic heavy-ion beams provided by the SIS18 synchrotron accelerator of GSI, Darmstadt. The essential feature of this approach consists in providing the fissile nucleus to be investigated as a projectile, either as a primordial nuclide directly from the ion source of the accelerator complex or as a secondary beam. Fission is induced by electromagnetic or nuclear interaction in a suitable target. By inverting the kinematics with respect to conventional fission experiments, in which the fission products of the target nucleus are measured, the fission products of the projectile nucleus appear with considerably higher kinetic energies, and thus the detection and identification of all fission products becomes feasible. The present contribution provides an overview on the new experimental possibilities offered by the installations of GSI. An overview on the experimental results on multimodal fission in the light actinides and on nuclide production cross sections in spallation and fragmentation reactions will be given.

These experiments do not directly respond to the requests on nuclide production in fission reactions from applications in nuclear technology and fundamental research, since only a limited number of key reactions could be studied due to the tremendous experimental effort. Therefore, a nuclear-reaction code has been developed on the basis of the body of measured data and which can be used for reliable predictions of nuclide production with different projectile and target nuclei and at different beam energies. As an important part of this code, a new fission model will be described in some detail. It is based on the powerful macroscopic-microscopic approach. In contrast to other purely theoretical descriptions, however, several critical ingredients, which cannot be predicted with the desirable accuracy, are extracted from the available experimental data.

\section{Experimental results on multimodal fission in the light actinides}

The secondary-beam facility at GSI was used to produce more than 70 different neutron-deficient actinides and pre-actinides by fragmentation of a ${ }^{238} \mathrm{U}$ beam at $1 \mathrm{~A} \mathrm{GeV}$ in a primary beryllium target. The fragmentation residues were sepa- 
rated and unambiguously identified event by event in atomic number $Z$ and mass number $A$ using the fragment separator FRS. These secondary beams impinged on a secondary lead target mounted at the exit of the FRS, where fission was induced by electromagnetic excitations and by nuclear collisions. The nuclear charges and the kinetic energy of both fission products were determined from the measurement of their energy loss and time of flight, see Figure 1. The energy loss was measured in a vertically subdivided ionisation chamber with a common cathode. The velocities of both fission residues were deduced by the time-offlight measurement from a plastic scintillator placed in front of the secondary target to a plastic-scintillator wall located 5 meters behind. The time-of-flight was used to correct the velocity dependence of the energy loss measured with the ionisation chamber and to determine the kinetic energies of the fission residues.
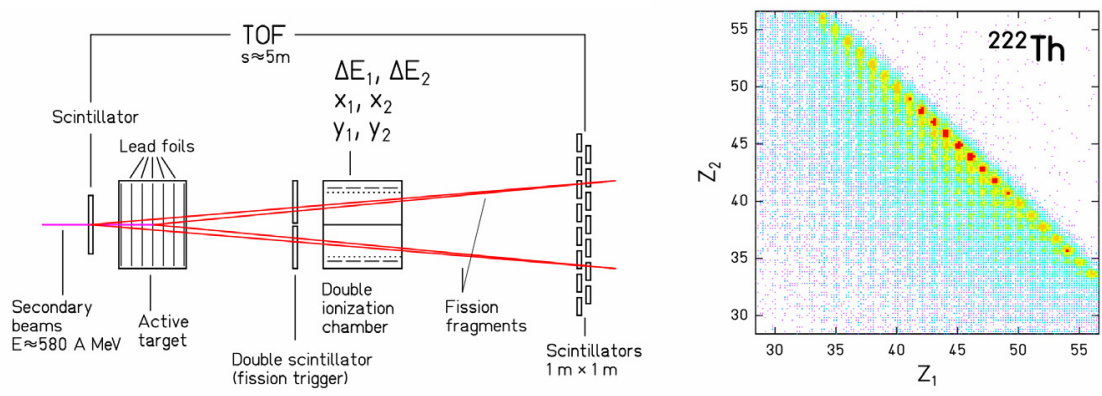

Figure 1: Left part: Schematic drawing of the set up for the fission experiment with secondary beams. Right part: Cluster plot of the nuclear charges of the two fission fragments from ${ }^{222} \mathrm{Th}$.

A selection on fission events induced by electromagnetic excitation was performed by requiring that the nuclear charges of the two fission fragments sum up to the number of protons in the secondary projectiles. A remaining fraction of nuclear-induced fission events was subtracted on the basis of the nuclear-charge spectrum from fission induced in the scintillator where the cross section for electromagnetic excitation is negligible. Electromagnetic excitations populate states at excitation energies around $11 \mathrm{MeV}$ with a width of a few MeV. In this energy range, shell effects still have a dominating influence on the nuclide distributions produced in fission. A detailed description of the experiment is given in ref. [1].

The elemental yields and the total kinetic energies of fission residues produced by electromagnetic excitation of secondary projectiles from ${ }^{205} \mathrm{At}$ to ${ }^{234} \mathrm{U}$ have been determined. In Figure 2, the elemental distributions of fission residues of 28 secondary beams between ${ }^{221} \mathrm{Ac}$ and ${ }^{234} \mathrm{U}$ are shown. The transition from symmetric fission in the lighter systems to asymmetric fission in the heavier ones is systematically covered for the first time. In the transitional region, 
around ${ }^{226} \mathrm{Th}$, triple-humped distributions appear with comparable intensities for symmetric and asymmetric fission.

The present knowledge on the global characteristics of the fission-fragment mass, respectively element distributions, in low-energy fission, acquired by all the experimental effort is summarized in Figure 3. The secondary-beam experiment has brought a systematic coverage of the transition from predominantly symmetric fission to predominantly asymmetric fission for fissioning systems around mass number 226. Previous investigations on these nuclei were rather hampered due to the lack of suitable target material. The large field of predominantly asymmetric fission in the actinides is also quite well covered by some 50 systems, by neutron- or light-charged-particle-induced fission of available target material or by spontaneous fission of nuclei produced in fusion reactions. The second region of bimodal fission with a transition back to symmetric mass distribution is just observed for the heaviest systems accessible to experiment, e.g. for ${ }^{258} \mathrm{Fm}$.

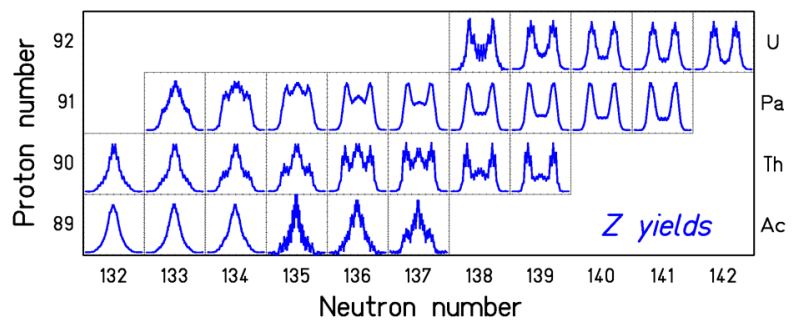

Figure 2: Measured fission-product element distributions in the range $Z=24$ to $Z=65$ after electromagnetic excitation of 28 secondary beams between ${ }^{221} \mathrm{Ac}$ and ${ }^{234} \mathrm{U}$ on a chart of the nuclides.

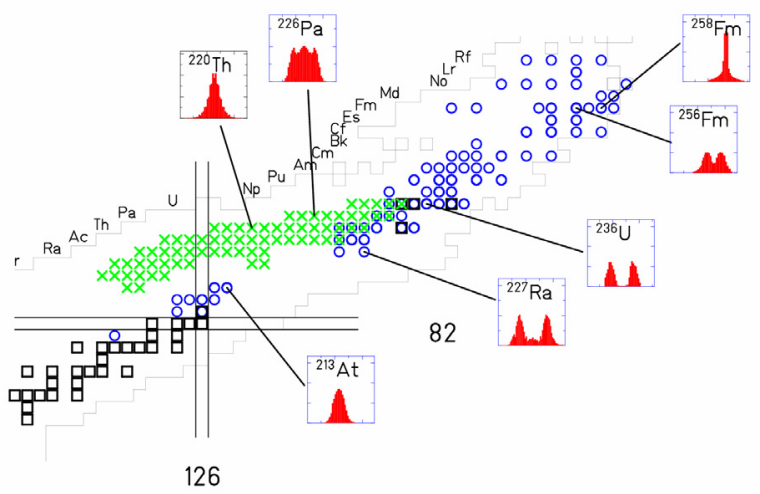

Figure 3: Present knowledge on mass and element distributions of fission products illustrated on the chart of the nuclides. Circles mark the fissioning systems investigated by conventional experiments in normal kinematics. Crosses denote the secondary beams investigated in the secondary-beam experiment performed at GSI in inverse kinematics. 


\section{Experimental results on nuclide production in spallation- and fragmenta- tion-fission reactions with ${ }^{238} \mathrm{U}$}

In another experimental configuration, the fragment separator FRS was used as a tool to separate and identify all reaction products in atomic number $Z$ and mass number $A$. However, this configuration is only applicable to beams of primordial nuclides. Due to the limited acceptance of the fragment separator in angle and magnetic rigidity only one fragment in limited magnetic-rigidity range can be detected at a time. However, the full nuclide production can be reconstructed by combining the results obtained with different magnetic fields. As a highly fissile projectile, ${ }^{238} \mathrm{U}$ with energies up to $1 A \mathrm{GeV}$ from the GSI accelerator facility was used to induce fission in electromagnetic excitations and nuclear collisions in different targets. In addition to the nuclide identification, see Figure 4, the recoil separator FRS also gives access to the reaction kinematics, and this information allows distinguishing fission events from other reaction channels, see

Figure 5.
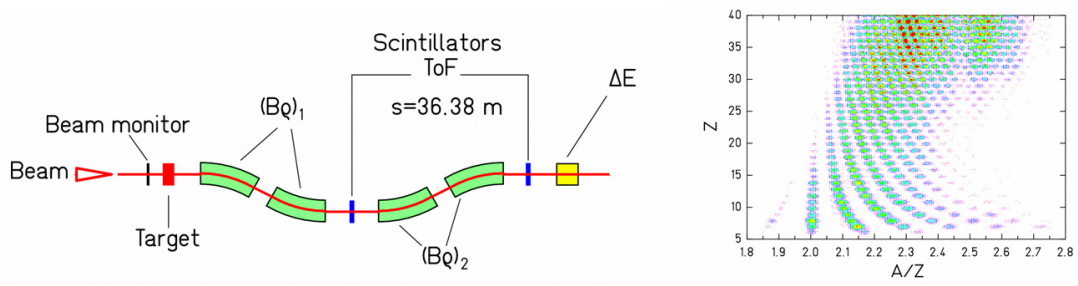

Figure 4: Schematic view on the fragment separator, operated as a magnetic spectrometer to identify the reaction products from the target (left part). Identification pattern of the light residues produced in the reaction ${ }^{238} \mathrm{U}(1 \mathrm{AGeV})+{ }^{1} \mathrm{H}$ (right part, from ref. [8]).
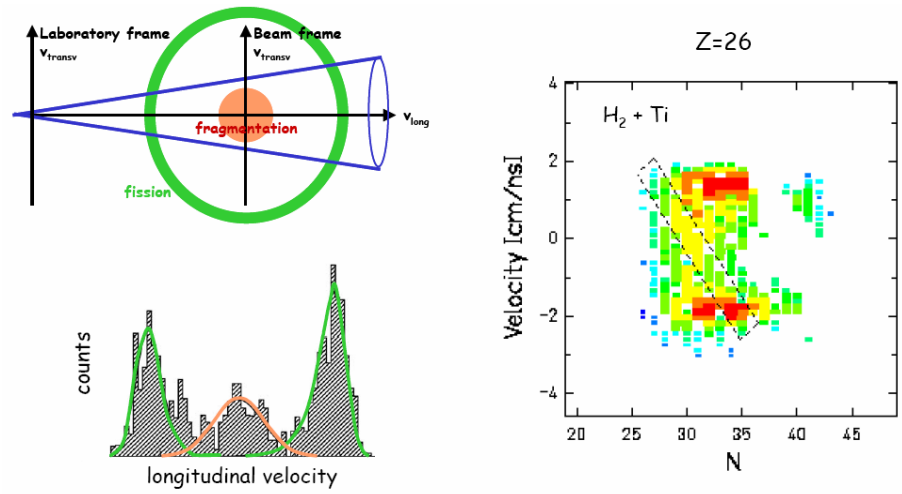

Figure 5: Demonstration of the kinematical cut due to the limited angular acceptance of the fragment separator. Residues emitted under small angles form different patterns: Fission residues appear in 
two humps from forward and backward fission, while evaporation residues appear in a single Gaussian-like distribution (from ref. [8]).

From the different studies on fission of ${ }^{238} \mathrm{U}$, e.g. $[2,3,4,5]$, we present the results of two experiments with a $1 A \mathrm{GeV}$ beam on a lead target [6] and on an hydrogen target $[7,8,9,10]$, respectively. A systematic overview on the full nuclide production in the reaction ${ }^{238} \mathrm{U}+{ }^{1} \mathrm{H}$ and on the fragmentation-fission residues in the reaction ${ }^{238} \mathrm{U}+\mathrm{Pb}$ is shown in Figure 6.
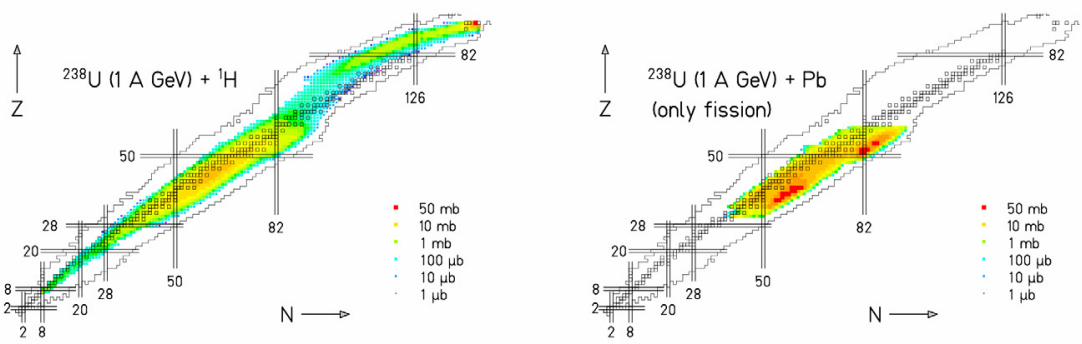

Figure 6: Measured nuclide production cross sections from the reactions ${ }^{238} \mathrm{U}(1 \mathrm{AGeV})+{ }^{1} \mathrm{H}$ (left part, from refs. $[7,8,9,11])$ and ${ }^{238} \mathrm{U}(1 A \mathrm{GeV})+\mathrm{Pb}$ (right part, from ref. [6]). For ${ }^{238} \mathrm{U}+{ }^{1} \mathrm{H}$, where spallation-evaporation and spallation-fission residues are rather separated in mass, all residues above $Z=6$ are shown. For ${ }^{238} \mathrm{U}+\mathrm{Pb}$, only fragmentation-fission residues are shown, because they strongly overlap with the spallation-evaporation residues. This experiment covered all elements between $Z=32$ and 59 .

One can observe that the nuclide distributions of the two reactions are rather different. The ${ }^{238} \mathrm{U}+{ }^{1} \mathrm{H}$ system mainly produces symmetric fission. The isotopes are situated close to beta stability. The ${ }^{238} \mathrm{U}+\mathrm{Pb}$ system mainly produces asymmetric fission, typical for low-energy fission in the actinide region. The main production is very neutron rich. This difference is explained by the different interactions leading to fission. While the proton, with its energy of $1 \mathrm{GeV}$ in the projectile frame, mostly interacts with the uranium projectile by violent nuclear collisions, leading to a broad excitation-energy distribution which reaches up to several hundred $\mathrm{MeV}$, the lead target nuclei also excite the uranium projectile by electromagnetic excitations with a large cross section at large impact parameters without any nuclear interaction. In this system, nuclear interactions with the lead target nuclei, which have a kinetic energy of $208 \mathrm{GeV}$ in the projectile frame, in most cases lead to multifragmentation or total disassembly of the projectile and thus do not contribute to fission.

\section{Semiempirical macroscopic-microscopic fission model}


In the following we present our model for the prediction of the nuclide distribution in fission. The model is imbedded in the dynamic de-excitation code ABLA [8], which considers the competition between evaporation of neutrons, light charged particles and intermediate-mass fragments on one side and fission on the other side. For excitation energies above the corresponding threshold also break-up and the simultaneous emission of several fragments is considered [12]. Fission is treated as a dynamical process, taking into account the role of dissipation in establishing quasi-equilibrium in the quasi-bound region by the implementation of a time-dependent fission-decay width [13]. When the system passes the fission barrier and proceeds to fission, it is characterised by mass and atomic number, excitation energy and angular momentum. It is the aim of our model to follow the descent from saddle to scission of the system and to predict the probability that its ends up in one of the many possible splits in $Z$ and $A$. We would like to mention that preliminary versions of this model have been presented previously $[14,15]$.

Most model descriptions of the fission process follow one of the following two roads: Either the evolution of the fissioning system is described with a purely theoretical model or the measured distributions of nuclides, kinetic energies and other observables are fitted by suitable functions with empirically determined parameters. The first road, i.e. the purely theoretical approach, is very challenging. Due to the complexity of the problem, any theoretical model has to introduce rather severe simplifications. In addition, one has to face the problem that the theoretical models are able to predict the relevant properties of a nuclear system only with a limited accuracy. This is obvious for the potential-energy surface in deformation space. Even in the nuclear ground state configuration, where the single-particle structure is generally studied very well, e.g. by nuclear spectroscopy, the empirical binding energy can only be reproduced with a standard deviation in the order of half an MeV. Such deviations are crucial for fission, e.g. a shift of $500 \mathrm{keV}$ in the ground-state binding energy modifies the spontaneous-fission half life by about 2 orders of magnitude [16]. Similar or even larger uncertainties are expected in the competition between different fission paths in low-energy fission. Thus, these models are very important for improving our understanding of the fission process, but their ability for quantitative predictions seems to be still rather limited. Following the second road, developing purely phenomenological models, one is able to reproduce measured data very well. However, the predictive power of phenomenological models for extrapolations far from explored regions is rather low due to the lack of the essential physics. We have chosen an intermediate approach based on physical models but using adjustments to experimental data. Of course, also our approach needs several simplifications in order to be tractable. We have tried to choose our simplifying assumptions in best accordance with present theoretical knowledge.

Starting with Fong [17] and followed later by Wilkins et al. [18], the statistical model has been taken as the basis of most fission models. Since the available 
phase space is a very important driving force of any process in nature, we consider the statistical model as the basis also of our model. In both previous models, it was avoided to consider dynamical effects by applying the statistical model at the scission configuration. This is a rather severe simplification, which is certainly not realistic. Depending on the relaxation times of the different collective degrees of freedom, some memory on previous configurations might be present. Therefore, we will discuss this point with some care.

First we start considering fission at high excitation energy, where shells and pairing correlations are negligible. Observed mass distributions of heavy fissioning nuclei above the Businaro-Gallone point (i.e. $Z^{2} / A>22$ ) from high excitation energies can well be described by a Gaussian distribution. This finding has been related to the available number of states above the potential energy as a function of mass asymmetry, since the potential can be approximated by a parabola near the minimum appearing at mass symmetry [19]. The second derivative $c_{\mathrm{A}}=\mathrm{d}^{2} U$ $/\left(\mathrm{d} A_{1}\right)^{2}$ of the potential as a function of the mass of one of the nascent fragments is related to the standard deviation $\sigma_{\mathrm{A}}$ of the mass distribution by the following relation:

$$
\sigma_{A}^{2}=\frac{T}{2 \cdot c_{A}}
$$

$T$ is the nuclear temperature, which is related to the excitation energy of the fissioning system $E=a T^{2}$. The coefficient $a$ is the level-density parameter.

This relation is a very important starting point of our model. Firstly, there exists a large body of experimental data on $\sigma_{\mathrm{A}}$ values, which provides the empirical data basis for a realistic prediction of fission mass distributions when structural effects are negligible. Secondly, the empirical result that the variance $\sigma_{\mathrm{A}}{ }^{2}$ is proportional to the nuclear temperature supports the validity of the statistical model. However, it is difficult to extract from these data, at which moment on the descent from saddle to scission the decision on the width of the mass distribution is taken. We can imagine two possible extremes. In one case, the phase space at the saddle point determines the mass asymmetry of the system, which is more or less frozen on a fast descent to scission. In the other case, the mass asymmetry degree of freedom adjusts very fast to the potential and thus it is finally determined at scission. Since a variation of the mass asymmetry is connected with a substantial transport of nucleons and, thus, the inertia should be large, we tend to support the first possibility. Following this idea, we take the systematics established in ref. [19] using the temperature at saddle in equation (1) for deducing the second derivative $c_{\mathrm{A}}$ of the potential from the experimental data. In fact, Itkis and Rusanov deduced a direct relation of $c_{\mathrm{A}}$ with the fissility parameter $Z^{2} / A$ of the fissioning nucleus. Thus, we have the first quantitative relation we use in our model to calculate the width of the mass distribution in case of sufficiently high excitation energies. 
Considering the fission process at lower excitation energies, our approach has to be substantially extended in order to include the appearance of fission channels. Early ideas for this concept are formulated in ref. [20]. Our experiments on multimodal fission in the light actinides [1] have considerably enriched our knowledge on the variation of structural effects in fission as a function of the nature of the fissioning system in terms of mass and atomic number. In particular, the gradual transition from double-humped mass distributions around ${ }^{238} \mathrm{U}$ to single-humped mass distributions around ${ }^{208} \mathrm{~Pb}$ has systematically been mapped. This experiment provides valuable information on the fission characteristic of specific fissioning systems at rather well defined excitation energies. We have used this specific information for developing the description of fission channels in our model, which should then be able to calculate the nuclide production in spallation-fission and fragmentation-fission reactions as the superposition of fissioning systems in many de-excitation processes from a large number of excited pre-fragments and a wide range of excitation energies.

Following the hypothesis that the mass asymmetry degree of freedom is essentially frozen at saddle, the probabilities for the population of the different fission channels should be decided at the outer saddle. Therefore, we assume that there is a direct correspondence between the shape of the potential at the outer saddle as a function of mass asymmetry and the population of the fission channels. The appearance of each fission channel is linked to a specific minimum in the massasymmetry dependent potential at the outer saddle. At this stage we empirically determine the depths and the widths of potential minima of the different fission channels by the weights and the widths of the corresponding components in the empirical nuclide distributions. For this purpose, we need to calculate the number of states available in the different potential minima. This time, the Fermi-gas level density is not realistic: We have to consider the level density in a configuration with a substantial shell effect. For this purpose, we use the analytical relation proposed by Ignatyuk et al. [21].

The description of Ignatyuk et al. requires the knowledge of the macroscopic potential. According to the previous discussion, we represent it by the parabolic potential deduced by Itkis et al. [20] from the widths of the mass distributions at high excitation energies.

As a starting point for the quantitative determination of the shell effects at the outer barrier, we consider the mass distribution of the fission fragments from ${ }^{238} \mathrm{U}(\mathrm{n}, \mathrm{f})$. As demonstrated in Figure 7, one obtains a rather consistent description, which reproduces the decrease of the relative population of the asymmetric fission channels with increasing excitation energy just by introducing two additional shells corresponding to the Standard 1 and Standard 2 fission channels, and by considering the washing out of the shell effects. Up to now, the shells at the outer barrier are formulated as a function of mass asymmetry. At this stage, we have a look to the results of two-centre shell-model calculations. They reveal that the shell effects at the outer barrier in ${ }^{238} \mathrm{U}$ are qualitatively similar to the shells in the separate fragments. It seems that the structure of the wave functions 
is quite similar all the way from the outer saddle to scission. This is not valid any more for more compact shapes, since the energetically favoured shape at the inner saddle is triaxial and mass-symmetric. Thus, we can profit from the investigations of Wilkins et al. [18] on the scission-point configuration, who stated that the most important shells behind the Standard 1 fission channel are $N=82$ and $Z=50$, while the Standard 2 fission channel is related to the $N \approx 90$ strongly deformed shell. Following these ideas, we attribute the two fission channels to the shells in the nascent heavy fragment mentioned above, while we neglect the influence of shell effects in the light fragment. From our adjusted parameters it appears that the spherical $N=82$ and $Z=50$ shells are considerably weaker than the shell effects we know from the ground-state masses around ${ }^{132} \mathrm{Sn}$. It might be assumed that the additional matter in the neck disturbs the symmetry of the nascent heavy fragment and reduces the shell gaps compared to the ideal spherical configuration we meet in ${ }^{132} \mathrm{Sn}$. The dominating appearance of the Standard 2 fission channel in ${ }^{238} \mathrm{U}(\mathrm{n}, \mathrm{f})$ seems to indicate that the deformed $N \approx 90$ shell, which appears less strong in the separate fragments, see the results of the shellmodel calculations in ref. [18], is less affected by the neck.

Once we have introduced the $N=82$ and $Z=50$ shells, we should consider the $N / Z$ degree of freedom. In order to include this degree of freedom, we replace the common Standard 1 shell effect, which was formulated as a function of mass asymmetry, by two contributions from the $N=82$ and the $Z=50$ shells. In fact, we use the known ground-state shell effects of the nuclei around ${ }^{132} \mathrm{Sn}$ with an adjusted reduction factor. This way, the shell effect at saddle responsible for the Standard 1 fission channel varies as a function of $N / Z$ of the fissioning nucleus. It is maximal for fissioning systems, which have the same $N / Z$ as ${ }^{132} \mathrm{Sn}$.
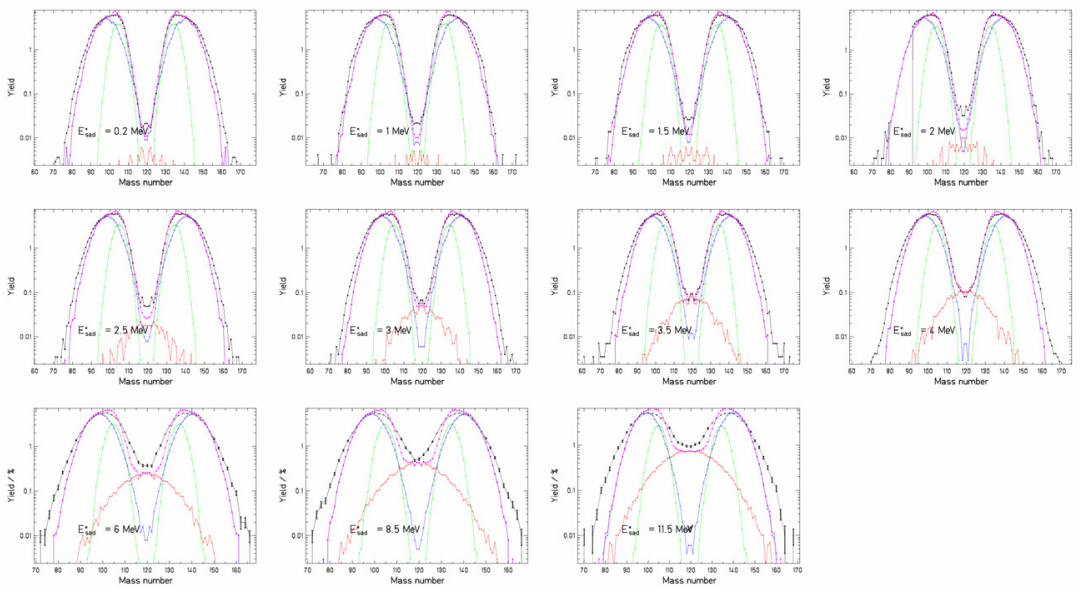

Figure 7: Calculated mass distributions (pink symbols) for neutron-induced fission of ${ }^{238} \mathrm{U}$ in comparison with experimental data (black symbols) $[22,23]$ for different values of the excitation energy above the fission saddle of the composite system ${ }^{239} \mathrm{U}$. The calculated individual contributions of the 
different fission channels are shown in addition: Standard 1 (green), standard 2 (blue), and superlong (orange).

After having adjusted the strengths and the widths of the three shells to the mass distributions of the system ${ }^{238} \mathrm{U}(\mathrm{n}, \mathrm{f})$, we are interested to check the predictive power of the model by applying it to other systems. We have chosen five isotopes around ${ }^{226} \mathrm{Th}$, were we have observed a triple-humped mass distribution. The good agreement with available experimental data in Figure 8 proves that a common description of ${ }^{239} \mathrm{U}$ and a series of thorium isotopes around ${ }^{226} \mathrm{Th}$ is possible by using the same set of parameters.

This result is not too surprising if the assumptions of our model are approximately valid. In particular, the shell effects at the outer saddle should have strong similarities to the shell effects in the separate fragments. Thus, they are only a function of $Z$ and $N$ of the nascent fission fragment, while they should be rather independent from the fissioning system.

Considering this success, we conclude that our model has a remarkable predictive power, once the parameters have carefully been deduced from experimental fission-fragment distributions.

We may formulate the most salient feature of our model as a rather peculiar application of the macroscopic-microscopic approach to nuclear properties. In our consideration of the properties of the fissioning system at the saddle configuration, we may attribute the macroscopic properties to the strongly deformed fissioning system, while the microscopic properties are attributed to the qualitative features of the shell structure in the nascent fragments. This way, the macroscopic and the microscopic properties are strongly separated, and the number of free parameters is independent from the number of systems considered.
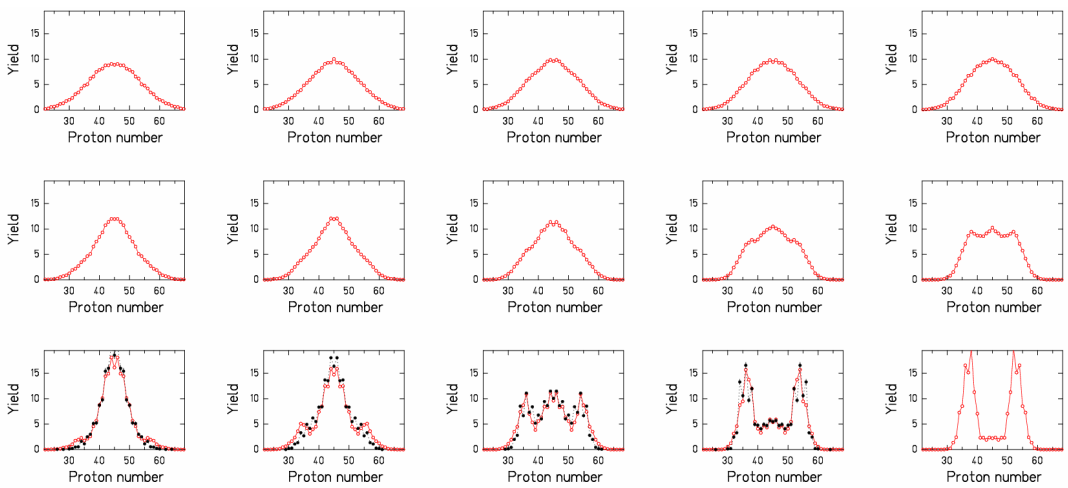

Figure 8: Calculated element distributions for ${ }^{220,223,226,229,232} \mathrm{Th}$ (from left to right) for excitation energies of 10, 20 and $60 \mathrm{MeV}$ (from bottom to top). The model parameters are the same as in Figure 7. Available experimental data from [1] are shown as black symbols for comparison. 
For completeness, we should mention that the model not only describes mass distributions but also considers the charge polarization in the nuclide production. Since there is only very little nucleon exchange necessary to exploit the full variation in $N / Z$ to be expected, we assume that the charge polarization is determined near scission. Quantitatively, the charge polarization is governed by the macroscopic contributions to the energy at scission [24] in most cases. Only the simultaneous influence of the $\mathrm{Z}=50$ and $\mathrm{N}=82$ shells lead to a rather important deviation from this trend for the Standard 1 fission channel, which tends to produce nuclides closer to the doubly magic ${ }^{132} \mathrm{Sn}$.

The full ABLA code, together with a suitable parameterization of the prefragments originating from nuclear-collision stage, was used to calculate the full nuclide distribution in the system ${ }^{238} \mathrm{U}(1 A \mathrm{GeV})+{ }^{1} \mathrm{H}[8]$ as shown in Figure 6. Better than comparing the two-dimensional production cross sections on a chart of the nuclides, which almost looks identical to the experimental result, we compare in Figure 9 the mean values and the FWHM of the isotopic distributions. This gives a more qualitative benchmark of the code. Obviously, the complex structural effects are very well reproduced. In particular, one can identify a strong contribution of the Standard 1 fission channel, responsible for the production of neutron-rich isotopes above tin and leading to an increase of the mean $N / Z$ ratio and an even stronger increase of the width of the isotopic distributions. The gradual decrease of the $N / Z$ ratio between $Z=60$ and $Z=70$ corresponds to the transition from spallation-fission to spallation-evaporation in this range. The data of the system ${ }^{238} \mathrm{U}(1 \mathrm{~A} \mathrm{GeV})+\mathrm{Pb}$ are reproduced with a similar quality. To obtain this good agreement, it is mandatory that the code calculates the fission fragments for a large number of fissioning systems in a large range of excitation energies in a realistic way.

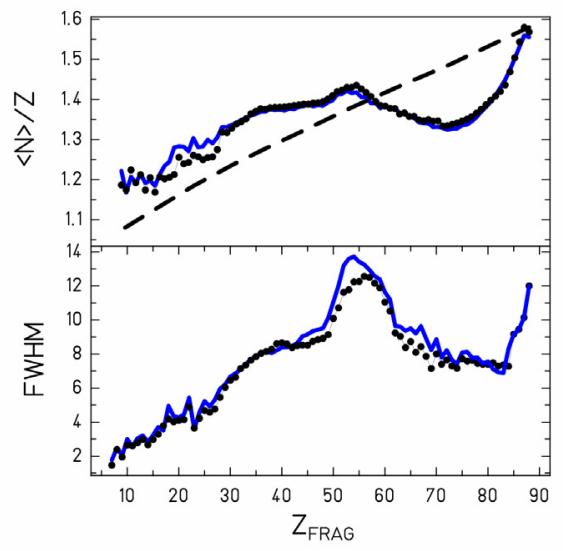

Figure 9: Comparison of the mean values and the FWHM of the isotopic distributions as a function of element number obtained in the reaction ${ }^{238} \mathrm{U}(1 \mathrm{~A} \mathrm{GeV})+{ }^{1} \mathrm{H}[8]$ in experiment (black symbols) 
and calculation (blue line). Contributions from spallation-evaporation and spallation-fission are included.

\section{Conclusion}

The systematic measurements performed at GSI with a novel experimental approach have considerably enriched our knowledge on the characteristics of nuclide production in fission. These results were used together with other experimental data to develop a new macroscopic-microscopic fission model with simplified assumptions on the dynamic of the system and an empirical adjustment of the potential-energy landscape of the fissioning system. The good reproduction of the nuclide distributions for a number of different fissioning systems over an extended mass range indicates that the assumptions of the model are rather realistic. Imbedded in a nuclear-reaction code, the model is able to reproduce the nuclide distribution in the spallation of ${ }^{238} \mathrm{U}$ quite well.

1. K.-H. Schmidt et al., Nucl. Phys. A 665, 221 (2000).

2. P. Armbruster et al., Z. Phys. A 355, 191 (1996).

3. C. Donzaud et al., Eur. Phys. J. A1, 407 (1998).

4 W. Schwab et al., Eur. Phys. J. A2, 179 (1998).

5. J. Pereira et al., Phys. Rev. C, in press.

6. T. Enqvist et al., Nucl. Phys. A 658, 47 (1999).

7. M. Bernas et al., Nucl. Phys. A 725, 213 (2003).

8. M. V. Ricciardi et al., Phys. Rev. C 73, 014607 (2006).

9. M. Bernas et al., Nucl. Phys. A 765, 197 (2006).

10. P. Armbruster et al., Phys. Rev. Lett. 93, 212701 (2004).

11. J. Taieb et al., Nucl. Phys. A 724, 413 (2003).

12. K.-H. Schmidt et al., Nucl. Phys. A 710, 157 (2002).

13. B. Jurado et al., Nucl. Phys. A 747, 14 (2005).

14. J. Benlliure et al., Nucl. Phys. A 628, 458 (1998).

15. K. Kruglov et al., Eur. Phys. J. A 14, 365 (2002).

16. Z. Patyk et al., Nucl. Phys. A 491, 267 (1988).

17. P. Fong, Phys. Rev. C 17, 1731 (1978).

18. B. D. Wilkins et al., Phys. Rev. C 14, 1862 (1976).

19. M. G. Itkis et al., Phys. Part. Nucl 29, 160 (1998).

20. M. G. Itkis et al., Sov. J. Nucl. Phys. 43, 719 (1986).

21. A. V. Ignatyuk et al., Sov. J. Nucl. Phys. 21, 255 (1975).

22. F. Vives et al., Nucl. Phys. A 662, 63 (2000).

23. C. M. Zöller, PhD thesis, Technical University Darmstadt, 1995

24. P. Armbruster, Nucl. Phys. A 140, 385 (1970). 